\title{
Party Role
}

National Cancer Institute

\section{Source}

National Cancer Institute. Party Role. NCI Thesaurus. Code C41108.

This object connects the party (organization or person) with the animal model. 